\title{
LRIS imaging spectropolarimeter at the W.M. Keck Observatory
}

\section{Robert Goodrich, Marshall Cohen}

Robert Goodrich, Marshall Cohen, "LRIS imaging spectropolarimeter at the W.M. Keck Observatory," Proc. SPIE 4843, Polarimetry in Astronomy, (14 February 2003); doi: 10.1117/12.458652

SPIE Event: Astronomical Telescopes and Instrumentation, 2002, Waikoloa, Hawai'i, United States 


\title{
The LRIS imaging spectropolarimeter at the W. M. Keck Observatory
}

\author{
Robert W. Goodrich ${ }^{a}$, Marshall H. Cohen ${ }^{b}$ \\ ${ }^{a}$ W. M. Keck Observatory, 65-1120 Mamalahoa Highway, Kamuela, HI 96743 USA \\ ${ }^{b}$ Palomar Observatory, California Institute of Technology, Pasadena, CA 91125 USA
}

\begin{abstract}
An optical-wavelength polarimetry module has been in use for the past ten years at the W. M. Keck Observatory. The module is used in conjunction with the LRIS imaging spectrograph. It provides either imaging polarimetry or spectropolarimetry across the full wavelength range of the instrument $(320-1100 \mathrm{~nm})$. The design, performance, and limitations of the polarimetry module are described, along with a sampling of science results.
\end{abstract}

Keywords: instrumentation, optics, polarization, spectropolarimetry

\section{INTRODUCTION}

Polarimetry has always been a niche subject in astrophysics, even though it provides some powerful tools that have broken new ground in a number of fields. One of the reasons for this is that polarimetry is "photon-starved." Imagine designing an imager or spectrograph that efficiently collects photons, only to throw out $99 \%$ of them in the end. This is in effect what is involved in measuring a polarization of $1 \%$. The trick is throwing out the right $99 \%$ !

One of the strengths of polarimetry lies in its ability to provide geometrical information about regions that are too small to image directly. In astronomy we are often limited by the limited spatial resolution that we have; this is one of the great driving forces behind orbiting telescopes like HST, and behind the push to develop ground-based adaptive optics and interferometry. Polarimetry can provide some information on even the smallest scales in astronomy.

The new generation of 6-10 m class telescopes has provided a major boost to polarimetry, providing large collecting areas feeding some sophisticated new instruments. For 10 years we have been using a polarimetry module in the LRIS (Low-Resolution Imaging Spectrograph) on the two $10 \mathrm{~m}$ Keck telescopes.

There are significant challenges to polarimetry in the modern era. Large primary mirrors are an increased challenge to coat uniformly, and inhomogeneities in the coatings can impart an instrumental polarization. Larger focal planes lead to larger optics. Or, if the scale in arcsec per pixel is held the same, smaller $\mathrm{f} /$ ratios are required, with the possible degradation in performance of the polarization optics.

Below we describe the LRIS polarimetry module in some detail, and follow this with a brief description of some of the science that has been produced with this instrument.

\section{LRIS OVERVIEW}

Originally a single-beam optical instrument that worked from 390-1100 nm, Keck's LRIS was designed from the beginning to allow its conversion to a dual-beam instrument by the addition of a dichroic and a blue arm. The original red arm allows for selectable filters, gratings and slits. A mirror can be used in the grating turret to provide an imaging 
capability. Space was made available between the slit and the spectrograph's field lens for removable modules. The only module built for LRIS so far is the polarimetry module that is the subject of this contribution.

In the summer of 1999 LRIS was upgraded to a dual-beam instrument, extending its UV coverage to the atmospheric cutoff (around $320 \mathrm{~nm}$ ).

\section{THE POLARIMETRY MODULE}

Although not included in the original LRIS design, the polarimetry module was designed and developed in parallel by the team of M. H. Cohen, R. W. Goodrich, and J. S. Miller. The module (Fig. 1) can be inserted and removed during the daytime, but not during the night (without an hour's worth of down time). It is self-contained as far as the polarization optics are concerned.

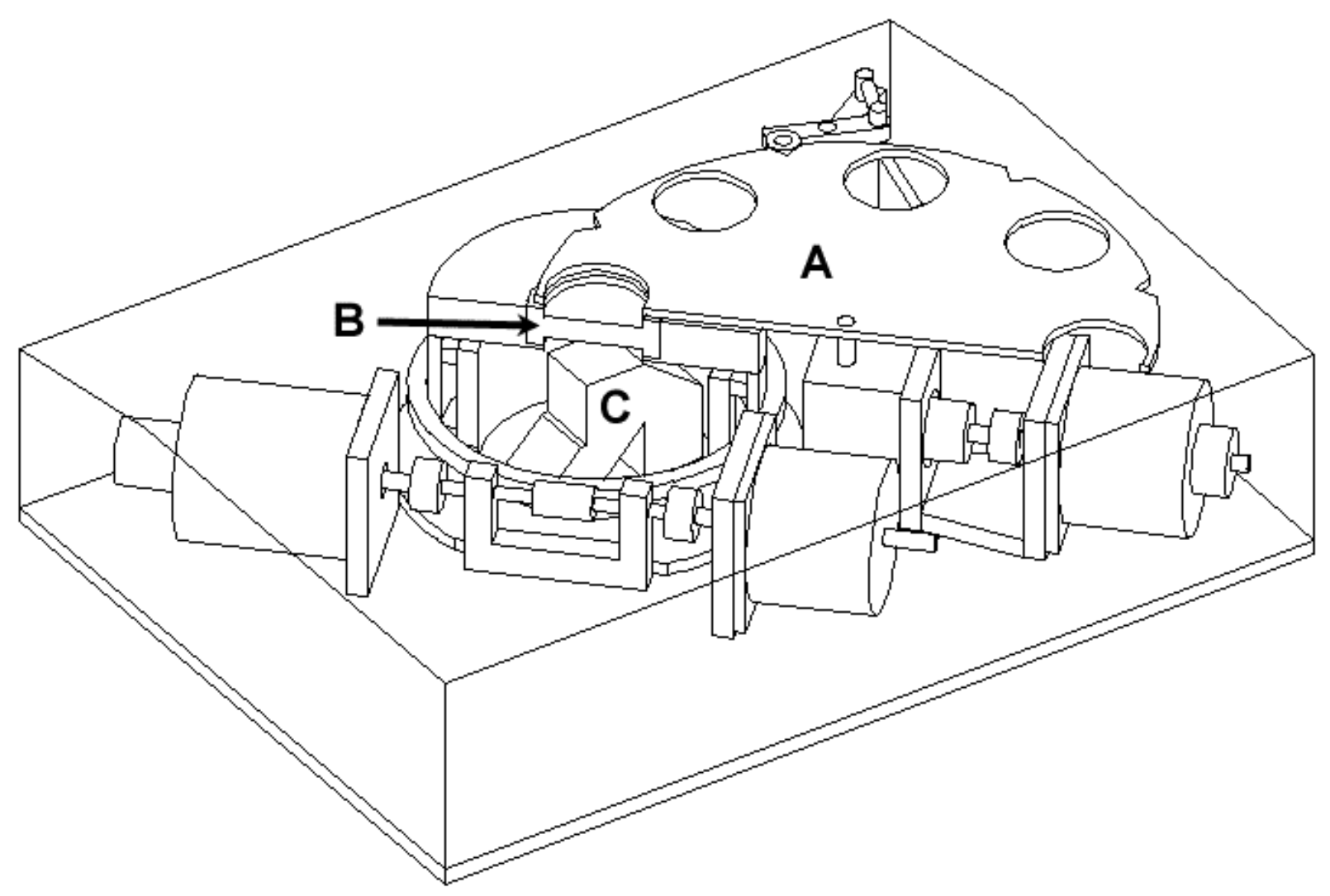

Figure 1. A cut-away drawing of the polarimetry module. Labeled are: (A) the calibration wheel, (B) the rotating wave plate, and (C) the polarizing beamsplitter. Light enters the module from above, passing through the left-most position of the calibration wheel, the rotating wave plate, and the top face of the beamsplitter. Two motors, an encoder, and various gears and couplings are also visible in the figure.

The relatively small vertical space available in LRIS to the removable modules limits the field of view of the polarimeter. The imaging polarimetry mode has a field of view of $35 \times 35 \mathrm{arcsec}$, and the spectropolarimetry mode allows for a 35 arcsec slit length. 
Linear and two methods of circular polarimetry are available. The full wavelength range of the dual-beam instrument is available by careful choice and design of the polarization optics.

\subsection{The Polarization Optics}

\subsection{Overview}

Directly below the LRIS slit is a "calibration wheel," which contains a quarter-wave plate, a linear polarizer for the optical wavelengths $(340-850 \mathrm{~nm})$, a linear polarizer for the near-IR $(750-1050 \mathrm{~nm})$, and opaque and clear positions. The optical polarizer is a Polaroid HNP'B polarizer, which will work efficiently down to $310 \mathrm{~nm}$. Below the calibration wheel is a rotating module holding a wave plate. Generally this contains a superachromatic half-wave plate, but the quarter-wave plate in the calibration wheel can be moved into this module for measuring circular polarization (section 7). Beneath the rotating wave plate is a polarizing beam splitter.

The optics and their performance are described in more detail in Paper $\mathrm{I}^{2}$ and Paper $\mathrm{II}^{3}$.

\subsection{The wave plates}

As mentioned above there are two wave plates available: a half-wave plate for linear polarimetry (and used for one of the two methods of measuring circular polarization), and a quarter-wave plate for circular polarimetry. Both are from B. Halle and both are "superachromatic" designs. The half-wave plate is a Pancharatnam design ${ }^{4}$ consisting of three quartz $/ \mathrm{MgF}_{2}$ "achromatic" half-wave plates. The combination of these three achromats provides a retardance within $1^{\circ}$ or $180^{\circ}$ from 325 to $1100 \mathrm{~nm}$.

The quarter-wave plate design is not that described by Pancharatnam. There is no simple analytical prescription for a superachromatic quarter-wave plate, but various search techniques over the design parameter space can let us find the design that maximizes the minimum efficiency over the 320-1100 nm wavelength range at a given (fixed) wave plate angle (see Paper II for details and performance).

\subsection{The polarization analyzer}

The analyzer is a polarizing beamsplitter designed to provide a single, continuous field of view, unlike some other designs that combine a "comb dekker" and an analyzer with limited beam separation to provide a large field of view. Our design avoids "edge effects" from the sides of the comb dekker and allows more straightforward observations of extended objects.

Our analyzer is a modified calcite "Glan-Taylor" polarizing beam splitter designed by RWG and made by Karl Lambrecht Corp (Figure 2). It is similar to the analyzers used in polarimeters at McDonald, Lick, and Palomar Observatories. A new method of compensating for the different optical path lengths of the two beams was designed by the authors and is implemented in the Keck version of this optic. A brief description is provided here; further details can be found in Paper II. 


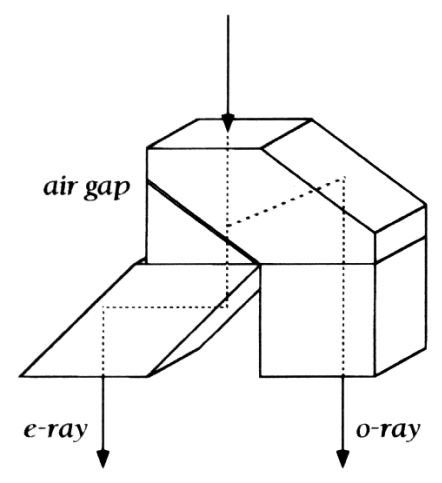

Figure 2. The polarizing beamsplitter used in the LRIS polarimetry module. The top two pieces are calcite, and the bottom two pieces Ultran 30. Light enters at the top face as indicated, and is split into the two senses of linear polarization at the air gap. The two Ultran 30 pieces are used to match optical path lengths of the two beams.

Light from the telescope enters the birefringent calcite crystal normal to the crystal's fast axis. Hence the two senses of linear polarization have different indices of refraction. At a tilted calcite/air interface the $o$-ray undergoes total internal refraction (due to its higher refractive index) while the $e$-ray passes through the interface with little reflective loss. A triangular piece of calcite crystal straightens the $e$-ray. Meanwhile, the once-reflected $o$-ray is reflected again by a second tilted calcite/air interface to make it parallel to, but offset from, the $e$-ray.

At this point the two rays have different optical path lengths, and we use a novel method of compensating for this. We use the extra horizontal space available in LRIS to match the path lengths, by sending the $e$-ray horizontal in glass with a $45^{\circ}$ reflection. This delays the $e$-ray. The beam must clear itself when it is reflected back to be parallel with the original beam, so there is a minimum horizontal distance we can use. Unfortunately this delays the $e$-ray too much!

We use a second block of glass underneath the $o$-ray to delay it the proper amount. In the end, the optical path lengths of both rays are nearly identical, and in fact are very close to the original optical path length of the instrument without any polarization optics. The glass used for the focus compensation is Ultran-30 from Schott. Ultran-30 was chosen as a UVtransmissive glass with a refractive index high enough to provide total internal reflection of the $\mathrm{f} / 15$ input beam at the $45^{\circ}$ interfaces in Fig. 2.

The analyzer splits the beams well over the entire wavelength range $(320-1100 \mathrm{~nm})$. Paper II describes the characteristics of the beamsplitter in more detail.

\section{DUAL-BEAM POLARIMETRY}

In general polarimeters can be separated into two classes: single-beam and dual-beam. The former requires at least two observations, separated in time, in order to determine a single Stokes parameter. The latter records both of these images simultaneously, hence avoiding time-variable effects like transparency, detector gain, seeing, slit losses, etc. For a single-beam instrument to be competitive, in terms of measurement precision, it typically must sample each of the two polarization senses at a rate faster than the changes in these parameters, in order to mitigate such effects. To provide even better calibration against systematic effects, dual-beam instruments typically use two pairs of images, as described below and elsewhere ${ }^{5}$. Finally, single-beam instruments discard one of the two polarization senses, dropping their efficiency by a factor of two over dual-beam instruments. 


\section{LINEAR POLARIMETRY}

Linear polarization can be described by the two Stokes parameters $Q$ and $U$. To measure one of these linear Stokes parameters we use the standard technique of recording both beams from the polarizing beamsplitter at each of two halfwave plate positions ${ }^{5}$. To measure $Q$ in the instrument's coordinate system we use half-wave plate positions of $0^{\circ}$ and $45^{\circ}$. To measure $U$ we use half-wave plate positions of $22.5^{\circ}$ and $67.5^{\circ}$.

Note that these Stokes parameters are in the instrument coordinate system, not the sky coordinate system. Also note that the superachromatic wave plates we use have a fast axis that varies as a function of wavelength. Hence the rotation from the instrument $(Q, U)$ coordinate frame to the sky frame is a function of wavelength. Fortunately for linear polarimetry, the correction is simply a matter of applying a wavelength-dependent rotation factor from the instrumental parameters to the sky (e.g. Paper I).

To measure the shape of this wavelength-dependent Stokes rotation, we observe a bright star through a polarizing element in front of the half-wave plate. These calibration polarizers provide high linear polarization at a constant angle. Then, to determine the zero-point of the polarization angle correction we observe a "polarized standard;" a star of known polarization such as can be found in various references ${ }^{6}$.

Linear polarization can be formed in a number of astrophysical processes. One of these is scattering. This has been used to great advantage in searching for regions hidden from direct view, but visible via scattering "mirrors" of dust or electrons. At Keck polarimetry is used almost exclusively on active galaxies showing scattering signatures.

Other sources of linear polarization are the strong magnetic fields in some white dwarfs (e.g. Zeeman splitting), or the synchrotron emission produced in active galaxies and some local "micro-quasars." Transmission through aligned dust grains in the interstellar medium can also be studied. This is, in fact, the dominant source of the polarization in the "polarization standards" mentioned above.

\section{CIRCULAR POLARIMETRY, METHOD 1}

The Keck polarimeter provides two methods for measuring circular polarimetry. In both cases a quarter-wave plate is used to convert circular polarization in the object to linear polarization that can be measured by the polarization analyzer. The circular Stokes parameter, $V$, requires only two observations for measurement.

With the first method, we use a fixed quarter-wave plate located in the calibration wheel of the polarimetry module, below the spectrograph's slit but above the rotatable half-wave plate and fixed polarization analyzer. The quarter-wave plate converts the circular polarization into linear polarization at a specific angle. By taking observations with the wave plate at two angles optimized to measure that particular angle of linear polarization, we measure the circular polarization in the object.

Note that with this method, unlike in linear polarimetry, a rotation of the fast axis of the quarter-wave plate relative to the ideal fast axis angle of the half-wave plate will result in a loss in measurement efficiency. This is also true of any deviation of the quarter-wave plate from the "optimum" angles mentioned in the preceding paragraph. The wavelengthdependence of the fast axis position of the half-wave plate is actually similar to the wavelength-dependence of the quarter-wave plate's fast axis, but the two do not perfectly compensate.

Another effect of the wavelength-dependence of the fast axis is leaking of the linear polarization from the object into the circular polarization measurements. This could explain the weak circular polarizations reportedly measured ${ }^{7}$ in active galaxies with high linear polarization.

This first method for measuring circular polarization also allows for linear polarimetry on the same or different objects by simply commanding the calibration wheel to an open position, removing the quarter-wave plate from the beam. This 
first method can be used on nights when both linear and circular polarimetry are planned, because swapping the wave plates is a daytime procedure (section 7).

\section{CIRCULAR POLARIMETRY, METHOD 2}

A second method can be used to measure circular polarimetry. In this case the half-wave plate is replaced by the quarterwave plate in the rotating unit. This is a daytime job, not something that can be done during the night. This is because of the large overhead (hours) involved in removing the instrument from the telescope, then the polarimetry module from the instrument, and swapping the delicate and expensive quarter-wave plate from the calibration wheel to the rotating turret, and then reinstalling everything.

Observations are made with the fast axis of the quarter-wave plate at $\pm 45^{\circ}$ to the fast axis of the beamsplitter. As with the first method for circular polarimetry, the wavelength-dependence of the quarter-wave plate's fast axis leads to a drop in efficiency in measuring circular polarization, and a leaking of linear polarization into the circular polarization measurement. This latter technique has never been used with the Keck polarimetry module, generally because the science projects have preferred to gather linear polarization information on the same objects or on other objects the same night.

Circular polarization can be used to study white dwarfs with magnetic fields, the interstellar medium, and magnetic A stars, for example.

\section{SCIENTIFIC EXAMPLES}

From the authors' own experience, we present here data from three polarimetry research projects.

\subsection{Broad-absorption line QSOs}

About $10 \%$ of all known quasars (QSOs, to be more rigorous) are observed to have strong, blueshifted absorption features in the high-ionization ions seen in the rest-frame ultraviolet. These broad absorption lines indicate that the absorbing gas is outflowing at high velocity, in some cases up to $0.2 c$. These objects are known as broad-absorption line QSOs, or BAL QSOs.

It is thought that BAL QSOs and normal QSOs represent the same class of objects, with the BAL QSOs seen from a special angle, one passing through a massive, high-velocity outflow. In non-BAL QSOs the flow is thought to be present, but we do not see it because it does not lie along our line of sight. This is an important concept, because it deals directly with the geometry and dynamics of the quasar phenomenon, and it has been important to investigate it in as many ways as possible.

It was noted some years ago ${ }^{8}$ that many BAL QSOs showed relatively high levels of broad-band linear polarization. Spectropolarimetry from 3-5 m telescopes ${ }^{9}$ provided the first clue that not only was the continuum polarized, but the light in the deep, blue-shifted absorption features that define the BAL QSO class can be highly polarized, moreso even than the continuum. However, with 4-m class telescopes, the observations were extremely difficult, and near-heroic efforts were required to obtain believable results.

The great light-gathering capability of LRIS and Keck I was used by Ogle et al. for a survey of BAL QSOs ${ }^{10}$ that really opened up the field. Figure 3 shows some of the spectropolarimetry from this reference. 


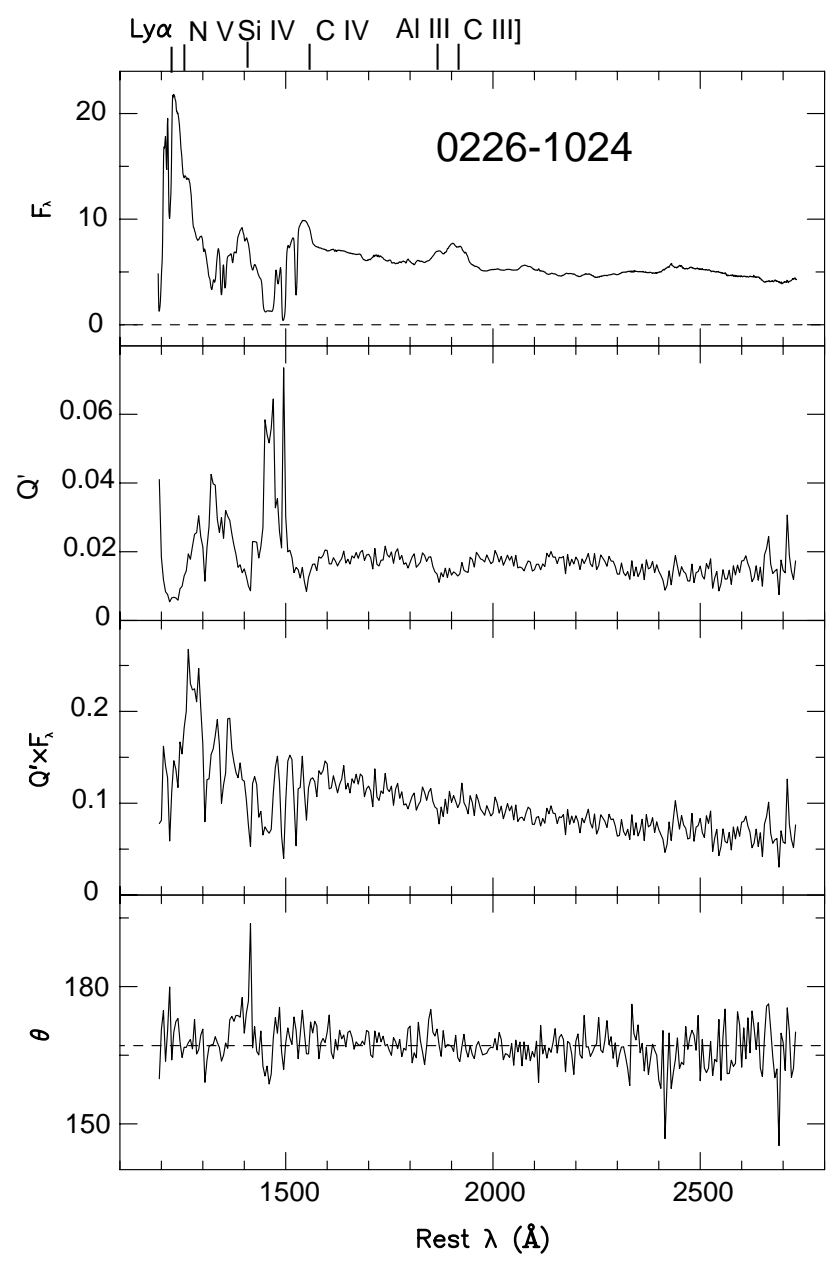

Figure 3. Keck linear spectropolarimetry of the broad-absorption line (BAL) QSO 0226-1024. $\mathrm{F}_{\lambda}$ is the total flux, as would be observed with a normal (nonpolarimetric) spectrograph. Note the emission features typical of QSOs and marked along the top axis. Also note the blue-shifted absorption features from C IV and Si IV, typical of BAL QSOs. Q' represents the fractional polarization, and shows polarizations up to $6 \%$ within the absorption features. The lower two panels show the polarized flux (the top two panels multiplied together) and the polarization position angle. The $\mathrm{x}$-axis is labeled with rest wavelengths, not observed wavelengths. The high redshift of these QSOs shifts these ultraviolet lines into the optical where ground-based telescopes can observe them.

Note in Fig. 3 how the polarization rises at wavelengths corresponding to the broad absorption troughs. This difference between absorption line and continuum polarizations, along with supporting evidence, shows that there are two separate paths for light to reach us from the central regions of the QSO. The direct view of the central source shows us the continuum, which is intrinsically low polarization. (We know this because non-BAL QSOs typically show low polarization.) However, at wavelengths where this light is heavily absorbed by intervening ions, we see highly polarized light. This light is scattered around most of the absorbing gas, becoming polarized in the scattering process. The scattered, polarized light partially fills in the deep absorption troughs of the directly viewed spectrum.

Further interpretation ${ }^{11}$ of the high polarizations seen in BAL QSOs implies that we are missing a large number of them in QSO surveys, because the obscuration of the direct view drops them below the survey detection limits. 


\subsection{High-redshift galaxies}

A second study ${ }^{12}$ concentrated on the nuclear radiation of high-redshift galaxies, with redshifts, $z \sim 2.5$. This redshift represents an epoch in the early Universe in which galaxies were first forming. These galaxies are faint, and long exposures with a large telescope are required to detect the polarization. We used up to 8 hours of exposure time with the Keck telescope. Some of the objects are highly polarized, up to $20 \%$, and the position angle of the polarization is often nearly perpendicular to the radio axis. As with low-redshift objects, this is taken as evidence of obscuring material, possibly a torus, in front of the nucleus. In analogy to the BAL QSOs, some of the nuclear light shining along the symmetry axis is scattered into our line of sight, and so is polarized.

The total flux spectra from this survey provided evidence of dramatic chemical evolution in the spheroids during this epoch. We further found a surprising correlation between the strengths of Ly $\alpha$ and $\mathrm{N} V$ emission lines and the degree of polarization (Fig. 4). Our interpretation is that this represents dispersal of the dust associated with the formation, early evolution, and chemical enrichment of the spheroid.

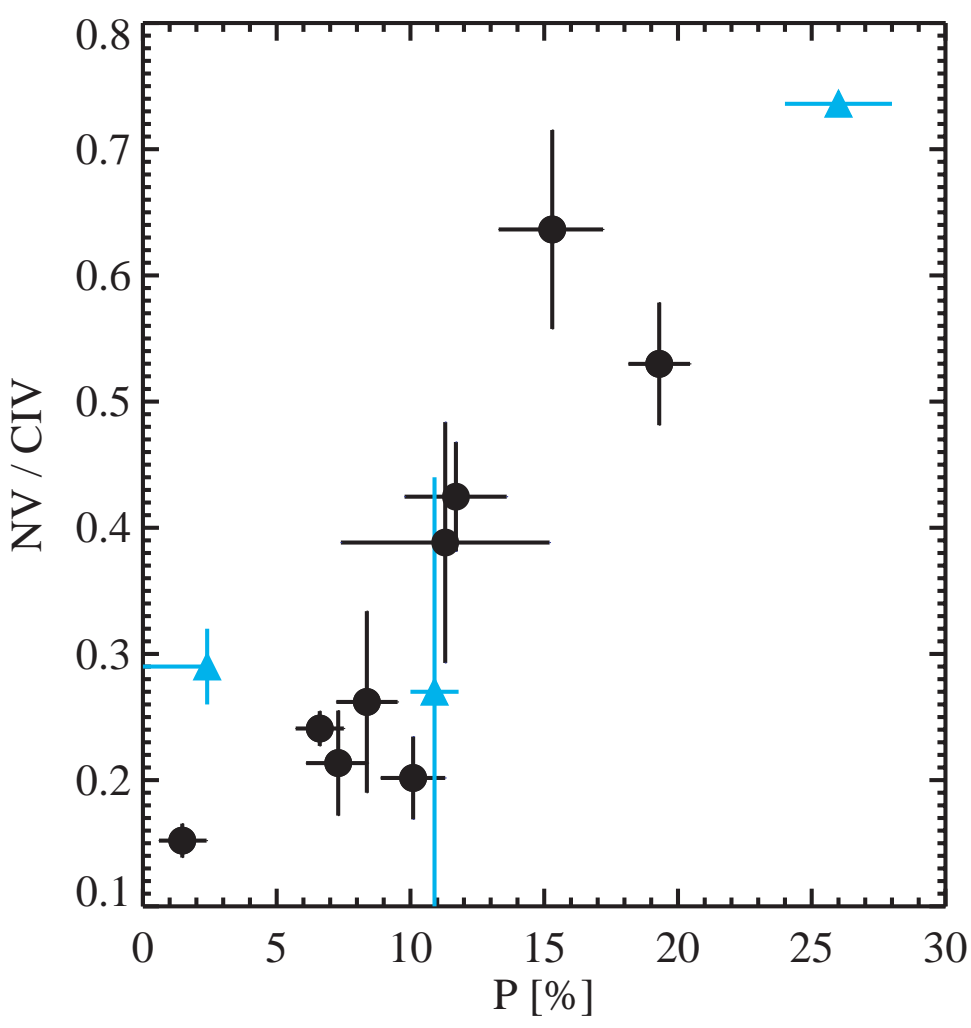

Figure 4. The correlation of polarization with $\mathrm{N}$ V/C IV emission line ratio seen in high-redshift radio galaxies.

\subsection{Magnetic fields in white dwarfs}

A third research project was the study of magnetic fields in white dwarfs. White dwarfs are the compact residual cores left after a normal star has collapsed near the end of its life. As the star collapses, any magnetic field in the gas can be concentrated to a very high level. White dwarfs can have magnetic fields of hundreds of MG. Small magnetic fields manifest themselves as a slight splitting of absorption lines into two or more components. The splitting is linear with field strength for weak fields. In total flux this small splitting leads to a broadening of the absorption line. This can be a subtle measurement to make and disentangle from other effects such as gravitational broadening. However, the different lines typically have different polarization characteristics, and with spectropolarimetry these can be more readily separated and studied. 
At higher magnetic field strengths, the linear response of the line wavelengths to field strength breaks down, and the wavelengths can reverse direction and even cross over the original, zero-field wavelength. This is shown in panel $\mathrm{C}$ of Figure 5, which shows the line wavelengths for the Balmer lines as a function of magnetic field strength. As an example, the $\mathrm{H} \alpha$ line lies at $6563 \AA(656.3 \mathrm{~nm})$ with zero magnetic field. At higher wavelengths five components are seen which vary between $5900 \AA$ and $8460 \AA$ at fields of $325 \mathrm{MG}$ (at the top of panel C).

The wavelengths at which these curves of magnetic field strength vs. wavelength become (temporarily) vertical are called "turning points." These are significant because at these points different parts of the atmosphere with slightly different magnetic field strengths contribute to the same wavelengths. If a significant portion of the atmosphere is near these field strengths, a deeper signature (in both polarization and total flux) is formed. In G 111-49, the Ha turning point just below $6800 \AA$ (one of the dotted lines) shows a significant polarization feature, and even a small feature in total flux (panel A). This turning point is sensitive to field strengths of $200 \mathrm{MG}$, and provides part of the evidence for a high magnetic field in this star. The data dramatically demonstrate how much more evident these features are in polarization compared to total flux, if the polarimetry has enough signal.

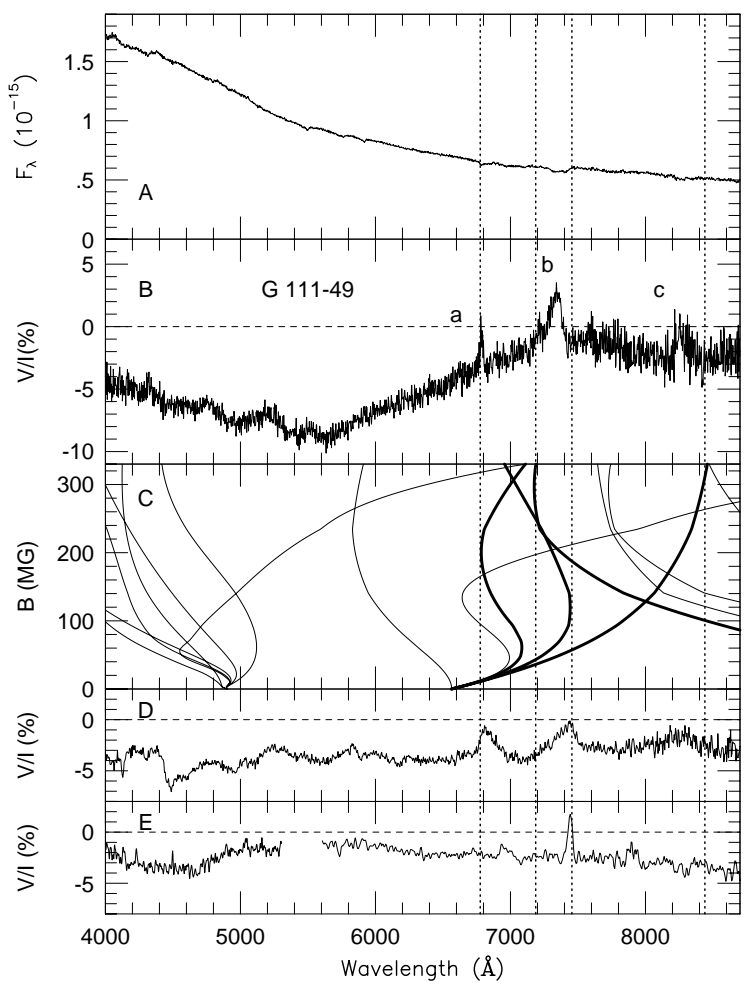

Figure 5. Spectropolarimetry of the DC white dwarf G 111-49. The total flux spectrum in the top panel shows a nearly featureless continuum spectrum; hence the classification as a DC white dwarf. However, circular spectropolarimetry in panel B shows a number of broad features that correspond to wavelengths called "turning points," which allow an estimate of the magnetic field strength on the star's surface for high magnetic fields. In the case of G 111-49 the spectropolarimetry indicates a magnetic field of $\sim 220 \mathrm{MG}$.

Putney ${ }^{13}$ conducted a survey of 52 objects classified as DC white dwarfs ("C" $\mathrm{C}$ " for continuum, implying that previous observations did not show absorption features). Because of the brightness of many of the survey targets, most of the data were from Palomar, but some, including the data shown above, used the LRIS polarimeter. 


\section{ACKNOWLEDGEMENTS}

The authors would like to acknowledge the many contributions of J. S. Miller and J. B. Oke to the design of the LRIS polarimetry module, and also H. Petrie, W. Schaal, and J. Cromer to the detailed design and construction. Some of the data presented herein were obtained at the W. M. Keck Observatory, which is operated as a scientific partnership among the California Institute of Technology, the University of California, and the National Aeronautics and Space

Administration. The Observatory was made possible by the generous financial support of the W. M. Keck Foundation.

\section{REFERENCES}

1. Oke, J. B., et al., “The Keck Low-Resolution Imaging Spectrometer,” PASP, 107, pp. 375-385, 1995.

2. Goodrich, Robert W., "High-Efficiency 'superachromatic' polarimetry optics for use in optical astronomical spectrographs," PASP, 103, pp. 1314-1322, 1991.

3. Goodrich, R. W., Cohen, Marshall H., and Putney, Angela, "Spectropolarimetry. II. Circular polarization optics and techniques," PASP, 107, pp. 179-183, 1995.

4. Pancharatnam, S., Proc. Indian Acad. Sci., A41, 137, 1955.

5. Miller, J. S., Robinson, L. B., and Goodrich, R. W., “A CCD spectropolarimeter for the Lick Observatory 3-meter telescope," in Instrumentation for Ground-Based Astronomy, ed. L. B. Robinson (New York, Springer), pp. 157-171, 1988.

6. Schmidt, Gary D., Elston, Richard, Lupie, and Olivia, L., "The Hubble Space Telescope northern grid of stellar polarimetric standards," $A J, 104$, pp. 1563-1567, 1992.

7. Valtaoja, L., Karttunen, H., Valtaoja, E., Shakhovskoy, N. M., and Efimov, Yu. S., "Optical circular polarization in two BL Lacertae objects?" A\&A, 273, pp. 393-396.

8. Stockman, H. S., Moore, Richard L., and Angel, J. R. P., “The optical polarization properties of 'normal' quasars," ApJ, 279, pp. 485-498, 1984.

9. Goodrich, Robert W., and Miller, Joseph S., "Polarization clues to the structure of broad absorption line quasi-stellar objects," ApJ, 448, pp. L73-L76, 1995.

10. Ogle, P. M., Cohen, M. H., Miller, J. S., Tran, H. D., Goodrich, R. W., and Martel, A. R., "Polarization of broad absorption Line QSOs. I. A spectropolarimetric atlas,” $A p J S, \mathbf{1 2 5}$, pp. 1-34, 1999.

11. Goodrich, Robert W., "On the fraction of broad absorption line quasi-stellar objects," ApJ, 474, pp. 606-611, 1997.

12. Vernet, J., Fosbury, R. A. E., Villar-Martín, M., Cohen, M. H., Cimatti, A., di Serego-Alighieri, S., and Goodrich, R. W., "Radio galaxies at $\mathrm{z} \sim 2.5$ : results from Keck spectropolarimetry," $A \& A, \mathbf{3 6 6}$, pp. 7-25, 2001.

13. Putney, A., "Surveying DC white dwarfs for magnetic fields," ApJ, 112, pp. 527-556, 1997. 